\title{
Reduction in Gastrointestinal Toxicity by Gastric Secretion Inhibitors during S-1 Monotherapy for Patients with Gastric Cancer
}

\author{
Yugo Chisaki, ${ }^{a, b}$ Satoshi Noda, ${ }^{b}$ Daiki Hira, ${ }^{b}$ Shin-ya Morita, ${ }^{b}$ Yoshitaka Yano, ${ }^{a}$ and \\ Tomohiro Terada*,b \\ ${ }^{a}$ Kyoto Pharmaceutical University; Kyoto 607-8414, Japan: and ${ }^{b}$ Department of Pharmacy, Shiga University of \\ Medical Science Hospital; Otsu, Shiga 520-2192, Japan. \\ Received January 10, 2014; accepted April 6, 2014
}

The purpose of this study was to retrospectively examine the effect of concomitant administration of gastric secretion inhibitors or gastrectomy on the frequency of gastrointestinal toxicity caused by 5-fluorouracil (5-FU) in gastric cancer patients receiving chemotherapy with S-1. In 62 gastric cancer patients treated with S-1 alone, data relating to the occurrence of gastrointestinal toxicity (diarrhea, vomiting, and nausea) and possible contributing factors were retrospectively collected, and logistic regression analysis was performed. Time-to-event data relating to the occurrence of gastrointestinal toxicity were also collected, and the effect of gastric secretion inhibitors on the time-to-event profiles was examined using a log-rank test. Logistic regression analysis suggested that the frequency of gastrointestinal toxicity was significantly reduced by the administration of gastric secretion inhibitors $(p=0.01 ;$ odds ratio, $0.197 ; 95 \%$ confidence interval (CI), 0.056-0.694) and that gastrointestinal toxicity correlated with the estimated glomerular filtration rate $(p=0.04$; odds ratio, $0.956 ; 95 \%$ CI, 0.916-0.997). The median time-to-event of gastrointestinal toxicity was $85 \mathrm{~d}$ for patients that received gastric secretion inhibitors, which was significantly different from the $42 \mathrm{~d}$ for patients that did not receive the inhibitors $(p=0.02$, log-rank test). No clear effect of gastrectomy was found in the present study. The prophylactic use of gastric secretion inhibitors in gastric cancer patients treated with S-1 may decrease and delay the occurrence of gastrointestinal toxicity.

Key words oteracil potassium (Oxo); S-1; gastric secretion inhibitor; gastrectomy; 5-fluorouracil

S-1 is an oral anti-cancer agent composed of tegafur (FT), 5-chloro-2,4-dihydroxypyridine (CDHP), and oteracil potassium (Oxo). ${ }^{1,2)}$ Five-year follow-up data of a recent phase III study, the Adjuvant Chemotherapy Trial of TS-1 for Gastric Cancer (ACTS-GC), confirmed that using S-1 as adjuvant chemotherapy for gastric cancer improves overall survival (OS) and relapse-free survival. ${ }^{3)}$ In the ACTS-GC study, patients who received fewer than the planned number of doses of S-1 because of the development of adverse events had worse outcomes than the patients who did not experience adverse events, ${ }^{3,4)}$ suggesting that the frequency of adverse events is an important factor in evaluating the efficacy of the drug.

S-1 includes Oxo to reduce the gastrointestinal (GI) toxicity associated with 5-fluorouracil (5-FU), which develops as a result of its phosphorylation by orotate phosphoribosyltransferase (OPRT) ${ }^{5)}$ Oxo reduces this cytotoxicity by inhibiting OPRT activity in the stomach and intestine, ${ }^{6,7)}$ as is demonstrated by the usefulness of Oxo in suppressing vomiting and diarrhea in dogs. ${ }^{89}$ ) In human studies, the frequency of GI toxicity was also reduced after treatment with S-1 compared to treatment with 5-FU alone. ${ }^{10,11)}$

Preclinical and clinical data have also indicated that Oxo is degraded by gastric acid, ${ }^{6,12)}$ and the area under the serum concentration curve $(A U C)$ of Oxo tended to increase with concomitant administration of proton pump inhibitors. ${ }^{12)}$ The $A U C$ and the maximum serum concentration of Oxo also tend to increase after total gastrectomy. ${ }^{13)}$ These findings suggest that the serum concentration of Oxo increases with the use of gastric secretion inhibitors or after gastrectomy. However, changes in such efficacy in patients have not been thoroughly examined. The purpose of the present study was to retrospec-

The authors declare no conflict of interest. tively examine the effect of concomitant administration of gastric secretion inhibitors or gastrectomy on the frequency of GI toxicity caused by 5 -FU in gastric cancer patients receiving S-1 chemotherapy.

\section{MATERIALS AND METHODS}

Collection of Patient Data This was a retrospective study and data were collected from patients who were treated for gastric cancer with S-1 chemotherapy alone from January 2010 until June 2013 at Shiga University of Medical Science Hospital (Otsu, Japan). The data collected included basic demographic information such as age, gender, aspartate aminotransferase (AST) level, estimated glomerular filtration rate (eGFR, $\mathrm{mL} \cdot \mathrm{min}^{-1} \cdot 1.73 \mathrm{~m}^{-2}$ ), gastrectomy status, concomitant use of gastric secretion inhibitors, and the use of gastric irritants. In this study, gastric secretion inhibitors were used at the same time with S-1 chemotherapy or at some days later for the palliation of stomachache. Patients receiving chemotherapy with agents other than S-1 were excluded. S-1 was administered for 4 weeks of every 6 weeks at a dose of $80 \mathrm{mg} / \mathrm{d}$ (body surface area (BSA), $<1.25 \mathrm{~m}^{2}$ ), $100 \mathrm{mg} / \mathrm{d}$ (BSA, 1.25 to $1.5 \mathrm{~m}^{2}$ ), or $120 \mathrm{mg} / \mathrm{d}\left(\mathrm{BSA},>1.5 \mathrm{~m}^{2}\right)$. However, the initial dose was reduced in some patients and the dose was also reduced during the treatment in the event of GI toxicity or for other reasons such as stomatitis. The types of GI toxicity considered in this study were the more commonly reported symptoms of diarrhea, vomiting, and nausea. These toxicities were evaluated according to the Common Toxicity Criteria of the National Cancer Institute Version 4.0, however the grading scores were not recorded and only the information of onset of these toxicities were available. This study was approved by the ethics committee of Shiga University of Medical Science Hospital. 
Data Analysis The differences of the characteristics between patients with GI toxicity and those without GI toxicity were compared using the $t$-test or chi-squared test. The occurrence of GI toxicity was determined, and the possible contributing factors were examined using logistic regression analysis. The colinearity among factors identified as significant in multivariate regression analysis was determined on the basis of the Spearman's rank correlation coefficient. The dependent variables examined were age, gender, AST level, eGFR, daily S-1 dose, gastrectomy, use of gastric secretion inhibitors, and use of gastric irritants. Missing values for an independent variable were replaced by the mean value of the patient population for that item. The odds ratio (OR) and $95 \%$ confidence interval (CI) were calculated for factors with significant coefficients in the logistic regression analysis. In addition, time-to-event curves for GI toxicity were generated using the Kaplan-Meier method for patient subgroups stratified according to the use of gastric secretion inhibitors, and the difference between the curves was tested using the log-rank test. The statistical significance level was set at $p<0.05$. The statistical software package, SPSS II Version 20.0, was used for all statistical analyses.

\section{RESULTS}

The basic characteristics of the 62 patients analyzed in this study are summarized in Table 1 . The initial S-1 dose was reduced to $c a$. $85 \%$ of their standard dose in patients with
GI toxicity $(30 / 45,67 \%)$ and those without GI toxicity (13/17, $77 \%$ ) ( $p=0.46$, chi-squared test). The S-1 dose was also reduced to $74.3 \%$ of their initial dose on average in 11 patients $(11 / 62,17.7 \%)$ during the chemotherapy treatment. The gastric secretion inhibitors were started at the same time or a few days later from the S-1 treatment, and had been concomitantly administered with S-1 until the termination of S-1 due to the occurrence of side effect or the ineffectiveness of S-1. Univariate analyses showed that eGFR and the use of gastric secretion inhibitors were statistically different between patients with GI toxicity and those without GI toxicity. The revelation rates of diarrhea, vomiting, and nausea were 53.3 (24/45), 31.1 $(14 / 45)$ and $28.9(13 / 45) \%$, respectively.

For further analysis, multivariate logistic regression using a model with all available covariates (full model) was performed, and the coefficients for eGFR and the use of gastric secretion inhibitors were significant (Table 2). Only these factors were incorporated into the subsequent model (final model) and re-analyzed. Table 3 shows the results of the final model analysis. In the final model analysis, gastrectomy was not determined to be a significant factor. The Spearman's rank correlation coefficient for eGFR and use of gastric secretion inhibitors was -0.04 , demonstrating that the colinearity was not significant $(p=0.77)$.

The logit function of the final model was as follows:

$$
\operatorname{logit}=2.20-0.045 \times \mathrm{eGFR}-1.63 \times x
$$

Table 1. Patient Characteristics

\begin{tabular}{|c|c|c|c|}
\hline & With GI toxicity $(n=45)$ & Without GI toxicity $(n=17)$ & $p$ Value \\
\hline Gender (male/female) & $35 / 10$ & $14 / 3$ & 1.00 \\
\hline Age (years) (mean, (S.D.)) & $65.4(11.0)$ & $67.8(11.5)$ & 0.47 \\
\hline AST (U/I) (mean, (S.D.)) & $25.3(7.57)$ & $16.9(10.7)$ & 0.58 \\
\hline eGFR $\left(\mathrm{mL} \cdot \min ^{-1} \cdot 1.73 \mathrm{~m}^{-2}\right)($ mean, (S.D.)) & $70.3(14.6)$ & $79.9(16.4)$ & 0.04 \\
\hline Gastric secretion inhibitors (use/no use) & $13 / 32$ & $11 / 6$ & 0.02 \\
\hline Omeprazole & 2 & 2 & \\
\hline Lansoprazole & 8 & 5 & \\
\hline Rabeprazole & 3 & 4 & \\
\hline Famotidine & 0 & 1 & \\
\hline Gastrectomy (yes/no) & $40 / 5$ & $15 / 2$ & 1.00 \\
\hline Treatment period of S-1 (d) (median, (S.D.)) & $238(245.7)$ & $315(280.6)$ & 0.46 \\
\hline Time to GI toxicity (d) (median, (S.D.)) & $56(63.4)$ & - & - \\
\hline Gastric irritant (use/no use) & $5 / 40$ & $1 / 16$ & 1.00 \\
\hline Loxoprofen & 3 & 1 & \\
\hline Etodolac & 2 & 0 & \\
\hline
\end{tabular}

Table 2. Results of Logistic Regression Analysis Using All Covariates (Full Model)

\begin{tabular}{|c|c|c|c|}
\hline Covariate & Regression coefficient & $p$ Value & Odds ratio $(\mathrm{OR})(95 \% \mathrm{CI})$ \\
\hline Age & -0.04 & 0.28 & $0.96(0.89,1.03)$ \\
\hline Gender (male: 1 , female: 0 ) & -1.76 & 0.08 & $0.17(0.24,1.25)$ \\
\hline AST level & 0.00 & 0.98 & $1.00(0.92,1.09)$ \\
\hline eGFR & -0.07 & 0.02 & $0.94(0.89,0.99)$ \\
\hline Daily dose of S-1 & 0.02 & 0.28 & $1.02(0.99,1.04)$ \\
\hline With gastrectomy (yes: 1, no: 0 ) & -0.23 & 0.85 & $0.80(0.08,8.00)$ \\
\hline Gastric secretion inhibitors used (yes: 1 , no: 0 ) & -1.93 & 0.02 & $0.15(0.03,0.70)$ \\
\hline Gastric irritant used (yes: 1 , no: 0 ) & 2.00 & 0.22 & $7.36(0.30,179.7)$ \\
\hline Intercept & 9.69 & 0.05 & - \\
\hline
\end{tabular}

CI: Confidence interval. 
Table 3. Results of Logistic Regression Analysis Using the Final Model

\begin{tabular}{lccc}
\hline \hline Covariate & Regression coefficient & $p$ Value & Odds ratio (OR) (95\% CI) \\
\hline eGFR & -0.045 & 0.04 & $0.956(0.916,0.997)$ \\
Gastric secretion inhibitors used (yes: 1, no: 0) & -1.63 & 0.01 & $0.197(0.056,0.694)$ \\
Intercept & 2.20 & 0.003 & - \\
\hline
\end{tabular}

CI: Confidence interval.

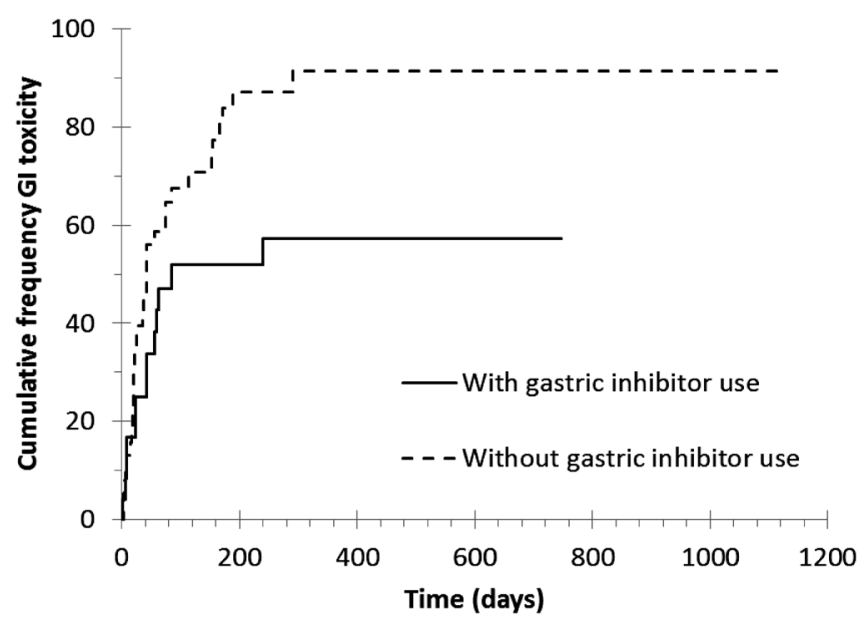

Fig. 1. Kaplan-Meier Plots for the Time-to-Event of Gastrointestinal Toxicity

The solid line and the dashed line represent patients with gastric inhibitor use $(n=24)$ and those without gastric inhibitor use $(n=38)$, respectively.

where $x$ takes a value of 1 (use of inhibitors) or 0 (no use of inhibitors). The ORs (95\% CIs) for the effects of eGFR and the use of inhibitors were $0.956(0.916,0.997 ; p=0.04)$ and $0.197(0.056,0.694 ; p=0.01)$, respectively. Since we found that the use of gastric secretion inhibitors significantly reduced the frequency of GI toxicity, we performed a time-to-event analysis of GI toxicity (Fig. 1). The median time-to-event, or time to occurrence of GI toxicity, in patients treated with gastric secretion inhibitors was delayed by $85 \mathrm{~d}$ compared to $42 \mathrm{~d}$ in patients not treated with inhibitors, resulting in significantly different time-to-event curves ( $p=0.02, \log$-rank test).

\section{DISCUSSION}

In this study, we sought to determine whether concomitant administration of gastric secretion inhibitors or gastrectomy alleviated GI toxicity caused by 5-FU in gastric cancer patients treated with the anti-cancer agent, S-1. As Oxo is degraded by gastric acid, ${ }^{6,12}$ the serum concentration of Oxo likely increases with the use of gastric secretion inhibitors, resulting in significantly reduced and delayed occurrence of GI toxicity in patients treated with inhibitors. In a post-marketing study of S-1 conducted in Japan, nausea, vomiting, and diarrhea were among the major toxicities observed during the first and second treatment courses. ${ }^{14)}$ These toxicities inconvenience patients, and thus, it is important to examine the contributing factors. Results from the present study suggest that the prophylactic use of gastric secretion inhibitors in gastric cancer patients treated with S-1 decrease and delay the occurrence of GI toxicity. Although it was not possible to separately evaluate the direct effect of gastric secretion inhibitors and the indirect effect via the decrease in Oxo, it was suggested that gastric secretion inhibitors is effective against GI toxicity in gastric cancer patients treated with S-1.

We also found that eGFR was a significant factor in causing GI toxicity. Renal function is an important consideration since renal dysfunction influences the frequency of GI toxicity. ${ }^{15)}$ A possible reason is the role of CDHP in S-1: CDHP is considered to increase the anti-cancer effect of 5-FU by inhibiting dihydropyrimidine dehydrogenase activity. ${ }^{16)}$ As CDHP is excreted into the urine, renal dysfunction may lead to an increased serum concentration of CDHP, followed by an increase in the 5-FU concentration. ${ }^{17}$ ) Thrombocytopenia and neutropenia were reported in patients with impaired renal function, ${ }^{18)}$ and GI toxicity likely increased with renal dysfunction.

Our study did not find a clear effect of gastrectomy on GI toxicity. This may be because of the small number of patients who did not undergo gastrectomy. Table 1 shows similar ratios of GI toxicity occurrence in patients who did and those who did not undergo gastrectomy, 0.73 (40/55) and $0.71(5 / 7)$, respectively. This suggests that GI toxicity occurred in this patient population at a high rate, irrespective of the performance of gastrectomy. Most of the patients $(55 / 62,89 \%)$ received segmental $(n=33)$ or total $(n=22)$ resection of stomach, and the degree of gastric acid secretion in these patients might be decreased compared with those without gastrectomy. However, gastric secretion inhibitors would be effective to prevent the degradation of Oxo as far as gastric acid is secreted from residual stomach.

In conclusion, we demonstrated that GI toxicity induced by S-1 was significantly decreased in patients who received gastric secretion inhibitors and in those with a high eGFR. Furthermore, gastric secretion inhibitors significantly delayed the occurrence of GI toxicity. The present study was based on a retrospective analysis of a limited number of patients and further studies may be required to validate our findings. Nonetheless, the results of the present study suggest that the prophylactic use of gastric secretion inhibitors in gastric cancer patients treated with S-1 may decrease and delay GI toxicity.

\section{REFERENCES}

1) Shirasaka $T$, Shimamato $Y$, Ohshimo H, Yamaguchi $M$, Kato $T$, Yonekura K, Fukushima M. Development of a novel form of an oral 5-fluorouracil derivative (S-1) directed to the potentiation of the tumor selective cytotoxicity of 5-fluorouracil by two biochemical modulators. Anticancer Drugs, 7, 548-557 (1996).

2) Shirasaka T, Shimamoto $Y$, Kato $T$, Fukushima M. Invention of a tumor-selective 5-fluorouracil derivative named S-1 by biochemical modulation of 5-fluorouracil. Gan To Kagaku Ryoho, 25, 371-384 (1998).

3) Sasako M, Sakuramoto S, Katai H, Kinoshita T, Furukawa H, Yamaguchi T, Nashimoto A, Fujii M, Nakajima T, Ohashi Y. Five-year 
outcomes of a randomized phase III trial comparing adjuvant chemotherapy with S-1 versus surgery alone in stages II or III gastric cancer. J. Clin. Oncol., 29, 4387-4393 (2011).

4) Sakuramoto S, Sasako M, Yamaguchi T, Kinoshita T, Fujii M, Nashimoto A, Furukawa H, Nakajima T, Ohashi Y, Imamura H, Higashino M, Yamamura Y, Kurita A, Arai K; ACTS-GC Group. Adjuvant chemotherapy for gastric cancer with S-1, an oral fluoropyrimidine. N. Engl. J. Med., 357, 1810-1820 (2007).

5) Houghton JA, Houghton PJ, Wooten RS. Mechanism of induction of gastrointestinal toxicity in the mouse by 5 -fluorouracil, 5 -fluorouridine, and 5-fluoro-2'-deoxyuridine. Cancer Res., 39, 2406-2413 (1979).

6) Nagayama S, Mita A, Masuda H, Toko K, Matsushita E, Yoshida K, Kawaguchi Y. Disposition of components of new anti-cancer drug S-1 (7): Metabolism of components of S-1 after administration to tumor bearing rats. Xenobio. Metabol. Dispos., 12, 645-655 (1997).

7) Shirasaka T, Shimamoto Y, Fukushima M. Inhibition by oxonic acid of gastrointestinal toxicity of 5-fluorouracil without loss of its antitumor activity in rats. Cancer Res., 53, 4004-4009 (1993).

8) Shirasaka T, Yamamitsu S, Tsuji A, Terashima M, Hirata K. Conceptual changes in cancer chemotherapy-biochemical modulation of 5-FU. Gan To Kagaku Ryoho, 27, 832-845 (2000).

9) Shirasaka T, Yamamitsu S, Tsuji A, Taguchi T. Conceptual changes in cancer chemotherapy: from an oral fluoropyrimidine prodrug, UFT, to a novel oral fluoropyrimidine prodrug, S-1, and low-dose FP therapy in Japan. Invest. New Drugs, 18, 315-329 (2000).

10) Huang J, Cao Y, Wu L, Liao C, He Y, Gao F. S-1-Based therapy versus 5-FU-based therapy in advanced gastric cancer: a metaanalysis. Med. Oncol., 28, 1004-1011 (2011).

11) Ohtsu A. Japanese nationwide post-marketing survey of S-1 in patients with advanced gastric cancer. Gan To Kagaku Ryoho, 33
(Suppl. 1), 52-56 (2006).

12) Scheulen ME, Saito K, Hilger RA, Mende B, Zergebel C, Strumberg D. Effect of food and a proton pump inhibitor on the pharmacokinetics of S-1 following oral administration of S-1 in patients with advanced solid tumors. Cancer Chemother. Pharmacol., 69, 753-761 (2012).

13) Kochi M, Fujii M, Kanamori N, Kaiga T, Aizaki K, Takahashi T, Takayama T. Effect of gastrectomy on the pharmacokinetics of S-1, an oral fluoropyrimidine, in resectable gastric cancer patients. Cancer Chemother. Pharmacol., 60, 693-701 (2007).

14) Nagashima F, Ohtsu A, Yoshida S, Ito K. Japanese nationwide postmarketing survey of S-1 in patients with advanced gastric cancer. Gastric Cancer, 8, 6-11 (2005).

15) Iwai M, Kimura M, Yoshimura T, Yasuda $T$. Side effects analyses in consideration of renal function for S-1-administered patients. Gan To Kagaku Ryoho, 38, 973-976 (2011).

16) Takechi T, Fujioka A, Matsushima E, Fukushima M. Enhancement of the antitumour activity of 5-fluorouracil (5-FU) by inhibiting dihydropyrimidine dehydrogenase activity (DPD) using 5-chloro-2,4dihydroxypyridine (CDHP) in human tumour cells. Eur. J. Cancer, 38, 1271-1277 (2002)

17) Ikeda M, Furukawa $H$, Imamura $H$, Shimizu J, Ishida H, Masutani S, Tatsuta M, Kawasaki T, Satomi T. Pharmacokinetic study of S-1, a novel oral fluorouracil antitumor agent in animal model and in patients with impaired renal function. Cancer Chemother. Pharmacol., 50, 25-32 (2002).

18) Yamanaka $T$, Matsumoto $S$, Teramukai $S$, Ishiwata $R$, Nagai $Y$, Fukushima M. Analysis of risk factors for severe adverse effects of oral 5-fluorouracil S-1 in patients with advanced gastric cancer. Gastric Cancer, 10, 129-134 (2007). 